\title{
ESTUDO DA INFLUÊNCIA DO MODO DE PREPARAÇÃO DO ORIFÍCIO PILOTO E DO MACHEAMENTO NA ANCORAGEM DOS PARAFUSOS PEDICULARES
}

\author{
STUDY OF THE INFLUENCE OF THE TYPE OF PILOT HOLE PREPARATION AND TAPPING ON \\ PEDICULAR SCREWS FIXATION
}

Helton luiz Aparecido Defino', José Roberto Benites Vendrame ${ }^{2}$, Antônio Carlos Shimano ${ }^{3}$, Frank Kandziora ${ }^{4}$

\section{RESUMO}

Foram realizados ensaios mecânicos com parafusos do sistema de fixação vertebral USIS para o estudo da influência do tipo de preparo do orifício piloto com sonda ou brocas e o macheamento do trajeto do orifício piloto, na resistência ao arrancamento dos implantes. Os parafusos foram inseridos em corpos de prova de madeira, poliuretana e osso bovino. O preparo do orifício piloto foi realizado com sondas e brocas de $3,5 \mathrm{~mm}$. Foram formados três grupos experimentais: I-perfuração com sonda, II-perfuração com broca e Ill-perfuração com broca e macheamento. Após a sua inserção nos corpos de prova foram realizados ensaios de arrancamento em máquina universal de teste. Foi observado aumento da resistência ao arrancamento dos implantes com a realização do orifício piloto com sondas e a diferença estatística foi significativa em relação ao preparo com broca e broca associada ao macheamento. Não foi observada diferença na resistência ao arrancamento dos parafusos com o macheamento do trajeto do orifício piloto.

Descritores: Fixação de fratura. Parafusos ósseos. Coluna vertebral.

Citação: Defino HLA, Vendrame JRB, Shimano AC, Kandziora F. Estudo da influência do modo de preparação do orifício piloto e do macheamento na ancoragem dos parafusos pediculares. Acta Ortop Bras. [periódico na Internet]. 2007; 15(4):200203. Disponível em URL: http://www.scielo.br/aob.

\section{INTRODUÇÃO}

A história da cirurgia da coluna vertebral está intimamente relacionada com a realização da artrodese vertebral e a utilização dos sistemas de fixação. O desempenho das funções mecânicas dos sistemas de fixação vertebral está relacionado com as propriedades mecânicas do sistema de fixação e com a ancoragem dos seus elementos de sustentação nas vértebras ${ }^{(1-4)}$.

A ancoragem do sistema de fixação nas vértebras está diretamente relacionada com as propriedades mecânicas da interface entre os implantes e o tecido ósseo. Os parafusos têm sido muito utilizados como componentes de ancoragem dos sistemas de fixação vertebral e as suas propriedades mecânicas estão relacionadas a vários fatores como a densidade mineral do tecido ósseo, o desenho e

\section{SUMMARY}

Mechanical assays were performed with screws of the USIS vertebral fixation system for the study of the influence of type of pilot hole preparation with probe or burr and tapping of the pilot hole pathway on pedicular screw pullout. The screws were inserted into wood, polyurethane and bovine bone test bodies. The pilot hole was prepared with probes and burrs of $3.5 \mathrm{~mm}$. Three experimental groups were formed: I -drilling with a probe, II - drilling with a burr, and III - drilling with burr and tapping. After screw insertion into the test bodies, pullout assays were performed with a universal test machine. Increased screw pullout resistance was observed when the pilot hole was drilled with a probe, with a statistically significant difference compared to preparation with a burr and with a burr in combination with tapping. No difference in screw pullout resistance was observed with tapping of the pilot hole pathway.

Keywords: Fracture fixation; Bone screws; Spine.
Citation: Defino HLA, Vendrame JRB, Shimano AC, Kandziora F. Study of the influence of the type of pilot hole preparation and tapping on pedicular screws fixation. Acta Ortop Bras. [serial on the Internet]. 2007; 15(4): 200-203. Available from URL: http://www.scielo.br/aob.

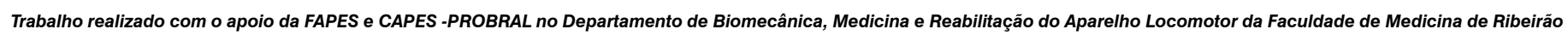
Preto da Universidade de São Paulo

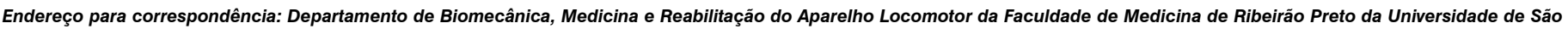
Paulo - Avenida Bandeirantes, 3900 - 11ㅇand - Campus Universitário - Ribeirão Preto/São Paulo - e-mail: hladefin@fmrp.usp.br

1. Professor Titular do Departamento de Biomecânica, Medicina e Reabilitação do Aparelho Locomotor da Faculdade de Medicina de Ribeirão Preto da Universidade de São Paulo

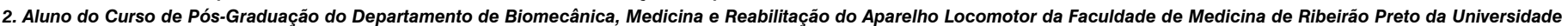
de São Paulo

3. Professor Doutor do Departamento de Biomecânica, Medicina e Reabilitação do Aparelho Locomotor da Faculdade de Medicina de Ribeirão Preto da Universidade de São Paulo 4. Chefe do Setor de Cirurgia da Coluna Vertebral do Departamento de Doenças do Aparelho Locomotor - Hospital Charitté - Berlin Head of Spine Center 


\section{MATERIAL E MÉTODO}

Foram utilizados no estudo corpos de prova de poliuretana, madeira e osso bovino. A espessura dos corpos de prova foi estabelecida com base na dificuldade para a realização dos testes de arrancamento dos implantes durante o estudo piloto. Os corpos de prova de poliuretana possuíam $27 \mathrm{~mm}$ de espessura, os de madeira 13 $\mathrm{mm}$ e os de osso bovino $17 \mathrm{~mm}$ de espessura. O corpo de prova de osso bovino era constituído da parte metafisária central e distal do fêmur, que era preparada com o auxílio de serra, removendo-se o osso cortical externo e confeccionando-se segmentos de osso esponjoso de $17 \mathrm{~mm}$ de espessura.

O implante utilizado no estudo foi o parafuso pedicular do sistema USIS (Ulrich), que possui $6 \mathrm{~mm}$ de diâmetro externo e 3,5 mm de diâmetro interno (Figura 1). Os parafusos foram implantados nos respectivos corpos de prova por meio da realização de orifício piloto de 3,5mm de diâmetro, que correspondia ao diâmetro interno (diâmetro da alma) do parafuso.

Após a realização do orifício piloto o parafuso era colocado no corpo de prova, transfixando-o e deixando $1 \mathrm{~cm}$ da sua extremidade distal exposta. Desse modo o número de rosca dos parafusos contidos nos corpos de prova era constante, e a extremidade distal exposta do parafuso era utilizada para a aplicação da força nos ensaios mecânicos de resistência ao arrancamento.

Os grupos experimentais foram formados de acordo com o modo de preparação do orifício piloto: grupo I - perfuração com sonda (3,5 mm), grupo II - perfuração com broca de 3,5 mm e grupo III - perfuração com broca de 3,5 mm e macheamento do orifício piloto. Os corpos de prova com os respectivos implantes foram submetidos a ensaios mecânicos realizados em máquina universal de ensaio (modelo EMIC ${ }^{\circledR}$, Brasil), ligada a computador e célula de carga de 200 Kgf, para a avaliação da resistência ao arrancamento dos implantes. O ensaio mecânico para a avaliação da resistência ao arrancamento foi realizado por meio de instalação de carga axial ao longo do eixo do parafuso a partir de sua extremidade, determinando a força máxima necessária para o seu arrancamento. A Figura 2 ilustra a representação gráfica fornecida pelo computador acoplado à máquina universal de ensaios e os valores aferidos em cada teste, uma representação no corpo de prova de poliuretana e outra no osso bovino, respectivamente.

Foram realizados 10 ensaios mecânicos nos grupos em que a madeira e a poliuretana foram utilizadas como corpos de prova e 15 ensaios no grupo em que foi utilizado osso bovino. Os resultados obtidos foram comparados por meio de estudo estatístico, tendo sido utilizado a análise de variância (ANOVA) e adotado o nível de significância $p \leq 0,05$. O teste de "post hoc de Bonferroni" foi utilizado para a comparação dos diferentes métodos de preparação do orifício piloto.

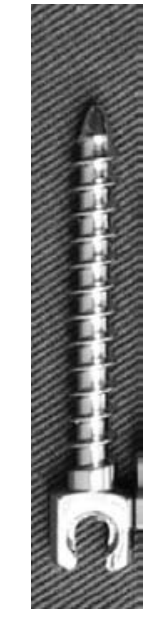

Figura 1 - Parafuso do sistema USIS (Ulrich) com $6 m m$ de diâmetro externo e $3,5 \mathrm{~mm}$ de diâmetro interno.

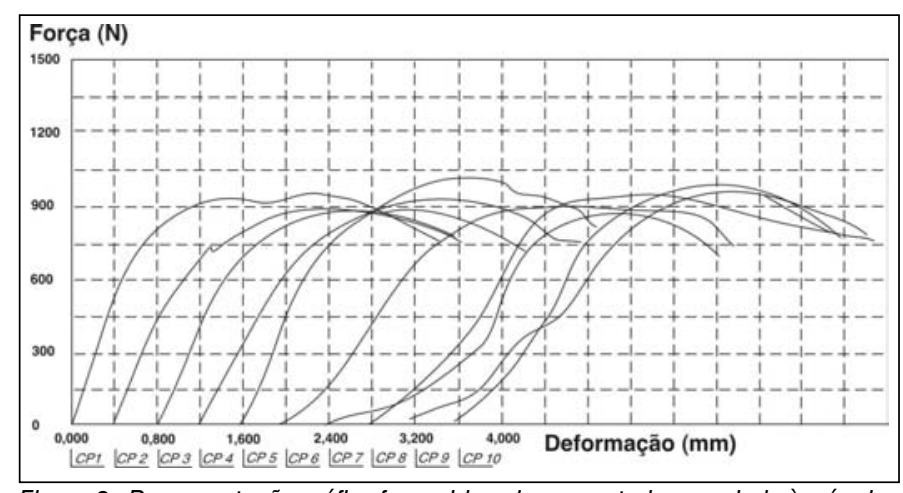

Figura 2 - Representação gráfica fornecida pelo computador acoplado à máquina universal de ensaios e os valores aferidos em cada ensaio mecânico.

\section{RESULTADOS}

Os valores obtidos nos ensaios mecânicos, considerando-se a média dos valores da força máxima de arrancamento dos implantes nos diferentes corpos de prova, estão representados nas Tabelas 1,2 e 3 e Figuras 3, 4 e 5.

\begin{tabular}{l|r|r|r|r}
\hline & $\begin{array}{l}\text { F o r c a a } \\
\text { M á x i m a } \\
\text { média (N) }\end{array}$ & $\begin{array}{l}\text { Desvio } \\
\text { padrão }\end{array}$ & $\begin{array}{l}\text { Coeficiente } \\
\text { de variação } \\
\text { (\%) }\end{array}$ & $\begin{array}{l}\text { N ú m e r o } \\
\text { de testes } \\
\text { realizados }\end{array}$ \\
\hline Sonda 3,5 mm & ${ }^{*} 942,6$ & 50,13 & 5,318 & 10 \\
\hline $\begin{array}{l}\text { Broca 3,5 sem } \\
\text { macheamento }\end{array}$ & 848,2 & 81,28 & 9,583 & 10 \\
\hline $\begin{array}{l}\text { Broca 3,5 com } \\
\text { macheamento }\end{array}$ & 883,2 & 85,5 & 9,681 & 10 \\
\hline
\end{tabular}

Tabela 1 - Valores da força máxima de arrancamento dos implantes nos corpos de prova de madeira. (*) Diferença estatística em relação aos demais valores.

\begin{tabular}{l|r|r|r|r}
\hline & $\begin{array}{l}\text { F o r c a a } \\
\text { M á x i m a } \\
\text { média (N) }\end{array}$ & $\begin{array}{l}\text { Desvio } \\
\text { padrão }\end{array}$ & $\begin{array}{l}\text { Coeficiente } \\
\text { de variação } \\
(\%)\end{array}$ & $\begin{array}{l}\text { N ú m e r o } \\
\text { de testes } \\
\text { realizados }\end{array}$ \\
\hline Sonda 3,5 & ${ }^{*} 73,45$ & 5,914 & 8,051 & 10 \\
\hline $\begin{array}{l}\text { Broca 3,5 sem } \\
\text { macheamento }\end{array}$ & 63,96 & 1,517 & 2,372 & 10 \\
\hline $\begin{array}{l}\text { Broca 3,5 com } \\
\text { macheamento }\end{array}$ & 62,24 & 3,332 & 5,353 & 10 \\
\hline
\end{tabular}

Tabela 2 - Valores da força máxima de arrancamento dos implantes nos corpos de prova de poliuretana. $\left({ }^{*}\right)$ Diferença estatística em relação aos demais valores.

\begin{tabular}{l|l|l|l|l}
\hline & $\begin{array}{l}\text { F o r c a } \\
\text { M á x i m a } \\
\text { média (N) }\end{array}$ & $\begin{array}{l}\text { Des vi o } \\
\text { padrão }\end{array}$ & $\begin{array}{l}\text { Coeficiente } \\
\text { de variação } \\
(\%)\end{array}$ & $\begin{array}{l}\text { N ú m e r o } \\
\text { de testes } \\
\text { realizados }\end{array}$ \\
\hline Sonda 3,5 & ${ }^{*} 1134$ & 243,0 & 21,43 & 15 \\
\hline $\begin{array}{l}\text { Broca 3,5 sem } \\
\text { macheamento }\end{array}$ & 903,9 & 213,2 & 23,59 & 15 \\
\hline $\begin{array}{l}\text { Broca 3,5 com } \\
\text { macheamento }\end{array}$ & 857,3 & 254,7 & 29,71 & 15 \\
\hline
\end{tabular}

Tabela 3 - Valores da força máxima de arrancamento dos implantes nos corpos de prova de osso bovino.(*) Diferença estatística em relação aos demais valores.

No grupo I (perfuração por meio de sonda de 3,5mm) a média dos valores da força máxima de arrancamento dos implantes foi de $942,5 \pm 50,13(\mathrm{~N})$ nos corpos de prova de madeira; 73,45 \pm $5,914(\mathrm{~N})$ nos corpos de prova de poliuretana e $1134 \pm 243$ nos corpos de prova de osso bovino.

No grupo II (perfuração com broca de 3,5mm) a média dos valores da força máxima de arrancamento dos implantes foi de 848,2 \pm 81,28 (N) nos corpos de prova de madeira; 63,96 $\pm 1,517(\mathrm{~N})$ nos corpos de prova de poliuretana e 903,9 \pm 213,2 nos corpos de prova de osso bovino.

No grupo III (perfuração com broca de $3,5 \mathrm{~mm}$ seguida de macheamento do orifício piloto) a média dos valores da força máxima de arrancamento dos implantes foi de 883,2 $\pm 85,5(\mathrm{~N})$ nos corpos de prova de madeira; $62,24 \pm 3,332(\mathrm{~N})$ nos corpos de prova de poliuretana e 857,3 $\pm 254,7$ nos corpos de prova de osso bovino. A comparação dos valores observados para o arrancamento dos implantes, considerando o modo de preparo do orifício piloto e os diferentes corpos de prova (madeira, poliuretana e osso) está representada por gráficos (Figuras 3, 4 e 5). Nos corpos de prova de madeira foi observado que a força máxima de arrancamento dos implantes foi maior no grupo I ( perfuração com sonda de $3,5 \mathrm{~mm})$ e apresentou diferença estatística $(p=0,03)$ entre o grupo II (perfuração com broca de 3,5mm). No entanto não foi observada 
diferença estatística entre o grupo I (perfuração com sonda de 3,5mm) e grupo o III (perfuração com broca de 3,5mm seguido de macheamento). Não foi também observada diferença estatística entre os valores do grupo II e III.

Nos corpos de prova de poliuretana foi observado que a força máxima de arrancamento dos implantes foi maior no grupo I (perfuração com sonda de $3,5 \mathrm{~mm}$ ) e foi observado diferença estatística $(p<0,001)$ entre o grupo I e os grupos II e III, cujos valores não apresentaram diferença estatística significativa.

Nos corpos de prova de osso bovino foi também observado que a força máxima de arrancamento dos implantes foi maior no grupo I (perfuração com sonda de 3,5mm) e foi observada diferença estatística $(p=0,006)$ entre os valores do grupo I e os valores do grupo II e III, cujos valores não apresentaram diferença estatística significativa.

A análise dos resultados mostra que a realização do orifício piloto com sonda com diâmetro igual ao diâmetro interno do parafuso aumentou a resistência ao arrancamento dos implantes quando comparado com a perfuração com broca de diâmetro também igual ao diâmetro interno do parafuso. O macheamento do trajeto do orifício piloto realizado com broca de diâmetro igual ao diâmetro interno do parafuso não produziu alteração estatística significativa da resistência ao arrancamento dos implantes.

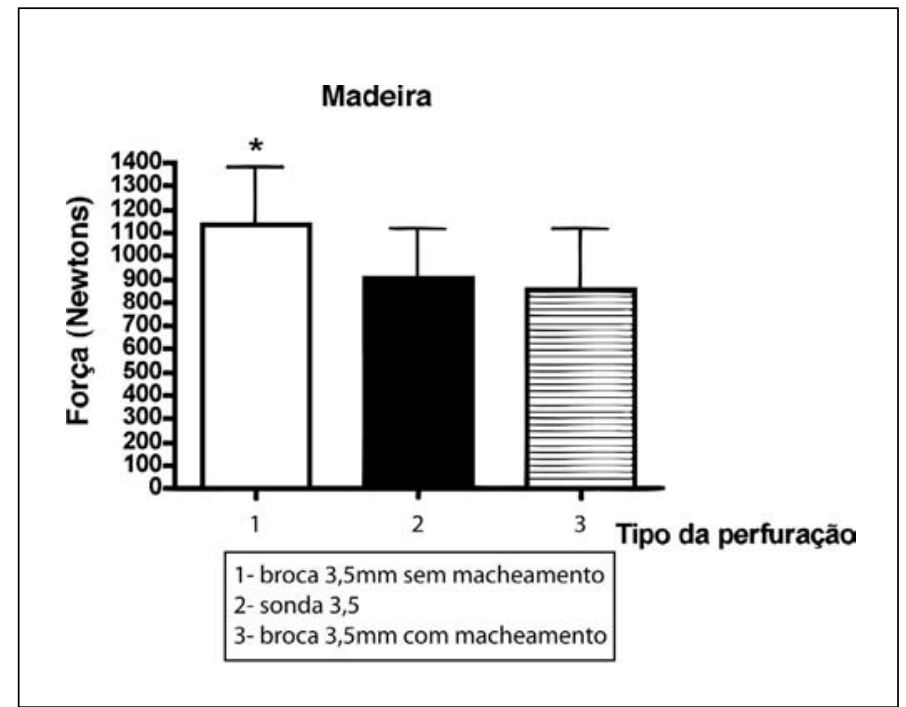

Figura 3 - A media da força máxima de arrancamento nos corpos de prova de madeira e as diferentes perfurações e preparo do orifício piloto.

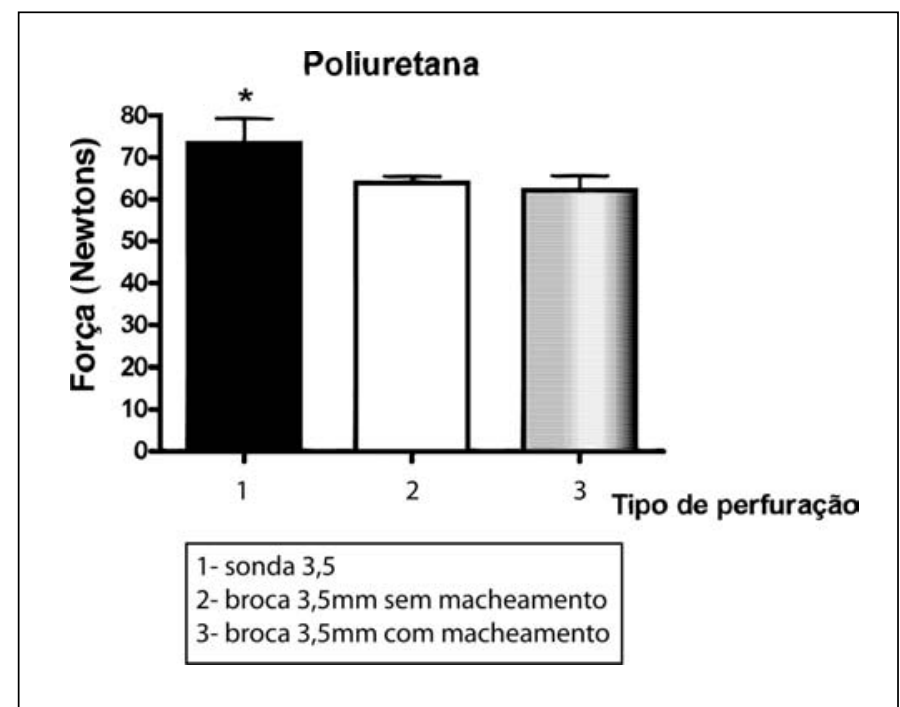

Figura 4 - A media da força máxima de arrancamento nos corpos de prova de poliuretana e as diferentes perfurações e preparo do orifício piloto.

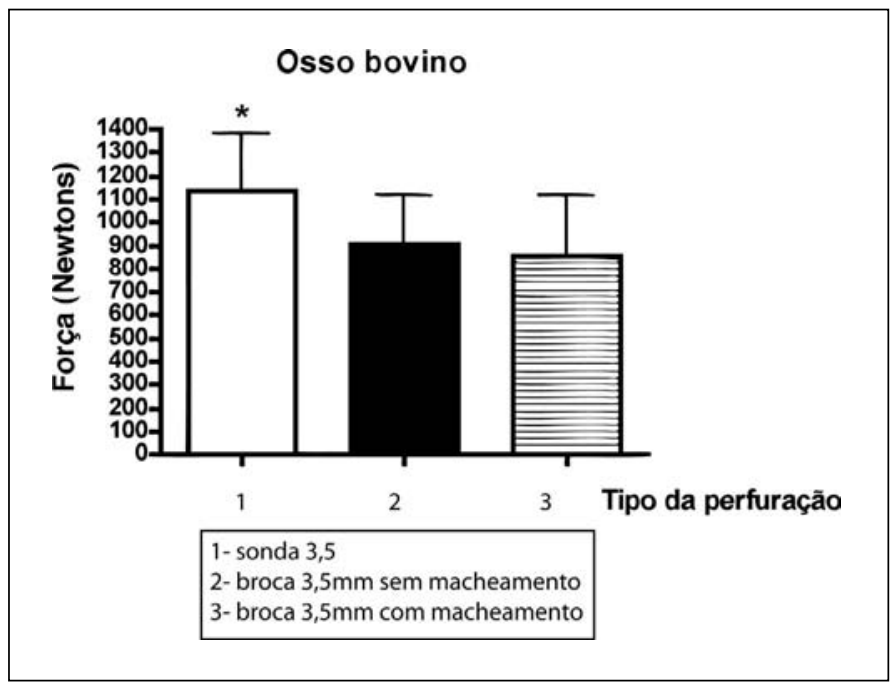

Figura 5 - A media da força máxima de arrancamento nos corpos de prova de osso bovino e as diferentes perfurações e preparo do orifício piloto.

\section{DISCUSSÃO}

A colocação dos parafusos no interior das vértebras é um passo técnico freqüente e de grande importância na cirurgia da coluna vertebral, pois a ancoragem dos parafusos na vértebra é a base para o desempenho da função biomecânica do sistema de fixação ${ }^{(4,5,8,9)}$ A técnica de inserção, o preparo do orifício piloto, o desenho do tipo de parafuso e a integridade e densidade do mineral do tecido ósseo influenciam o grau de fixação do parafuso no tecido ósseo, e desse modo a estabilidade do sistema de fixação e o resultado final do tratamento ${ }^{(8,10-12)}$.

A realização do orifício piloto está relacionada com muitas variáveis, destacando-se o seu diâmetro, o modo de preparo e o macheamento do seu trajeto ${ }^{(6,10,12-16)}$. A realização do orifício piloto permite a orientação e a definição prévia do trajeto e comprimento do parafuso, que devido às importantes estruturas vasculares e nervosas relacionadas com a anatomia da vértebra, aumentam a segurança do procedimento cirúrgico ${ }^{(6,11)}$

A resistência dos parafusos aos momentos fletores está relacionada com o seu diâmetro interno, e a sua resistência ao arrancamento ao diâmetro externo do parafuso, diâmetro da rosca, profundidade e desenho ${ }^{(5,6,8,15,16)}$. A resistência ao arrancamento é proporcional ao volume de osso entre as roscas do parafuso(5,8, 15-17).

Observamos em nossos ensaios que a utilização da sonda para a realização do orifício piloto aumentou a resistência dos parafusos ao arrancamento comparado com a utilização de brocas para a perfuração do orifício piloto. A utilização de sondas provocaria a compressão e compactação do osso esponjoso da vértebra ao redor do implante, aumentando a sua resistência ao arrancamento. A realização do orifício piloto com brocas provoca a remoção do tecido ósseo, reduzindo a resistência ao arrancamento do parafuso. A integridade do tecido esponjoso afeta a relação entre o torque de inserção e a resistência ao arrancamento(6,14-16,18) . No entanto, essa influência do tipo de preparo do orifício piloto não foi observada nos relatos de Daftari et al. ${ }^{(14)}$ e George ${ }^{(19)}$, que não observaram diferença na resistência ao arrancamento dos implantes com o preparo do orifício piloto com brocas ou sondas.

O macheamento do trajeto do orifício piloto tem sido relatado como um fator para a diminuição da resistência dos parafusos ao arrancamento ${ }^{(5,6,14,19)}$. No entanto, não observamos diferença da realização do macheamento na resistência ao arrancamento dos implantes nos ensaios realizados nos diferentes corpos de prova que estão de acordo com os relatos de Ronderos et al. ${ }^{(20)}$. O macheamento tem sido descrito com relacionado com a resistência ao arrancamento dos implantes e de utilização desvantajosa nos materiais moles, resultando em redução da resistência ao arranca- 
mento, devido às alterações estruturais que provoca na estrutura do osso esponjoso da vértebra(5). Carmouche et al. ${ }^{(13)}$ relataram a diminuição da resistência ao arrancamento dos implantes com o macheamento do trajeto do orifício piloto em vértebras lombares com osteoporose. Os corpos de prova que utilizamos em nosso estudo não simularam a condição da osteoporose, o que poderia explicar a não observância do efeito negativo do macheamento do trajeto do orifício piloto. Existem relatos de que o macheamento diminuiria a resistência ao arrancamento dos implantes nos ossos osteoporóticos, mas seria menos relevante nos ossos normais. $(5,12,21,20)$

O modelo de ensaio que realizamos foi delineado para a simples simulação da força necessária para o arrancamento dos implantes, e embora não reproduza as condições fisiológicas da aplicação das forças sobre os sistemas de fixação, permite a comparação e avaliação confiável dos parâmetros estudados. A resistência ao arrancamento dos implantes é um fenômeno complexo e multifatorial, e está relacionado a muitos fatores como o desenho dos parafuso, tipo e diâmetro da rosca do parafuso, densidade do osso e reações biológicas (reabsorção e remodelamento) que ocorrem no osso adjacente ao implante ${ }^{(5,12,17,19,22)}$.

\section{CONCLUSÕES}

O preparo do orifício piloto com a utilização de sondas aumentou a resistência ao arrancamento dos parafusos em todos os ensaios mecânicos realizados nos corpos de prova de madeira, poliuretana e osso bovino, comparado com os valores observados com a utilização de brocas para o preparo do orifício piloto. O macheamento do trajeto do orifício piloto perfurado com brocas não alterou a resistência ao arrancamento dos implantes utilizados nesse ensaio mecânico de arrancamento.

\section{REFERÊNCIAS BIBLIOGRÁFICAS}

1. Chen SI, Lin RM, Chang $\mathrm{CH}$. Biomechanical investigation of pedicle screw-vertebrae complex: a finite element approach using bonded and contact interface conditions. Med Eng Phys. 2003; 25:275-82.

2. Edwards CC, Garfin SR, Melkerson MN, Mishra NK, Winter RB, Yuan HA. Symposium: lumbar spine fixation--the pedicle screw controversy. Contemp Orthop. 1994: 29:439-54

3. Simmons JW, Andersson GB, Russell GS, Hadjipavlou AG. A prospective study of 342 patients using transpedicular fixation instrumentation for lumbosacral spine arthrodesis. J Spinal Disord. 1998; 11:367-74.

4. Defino HL, Vendrame JR. Role of cortical and cancellous bone of the vertebral pedicle in implant fixation. Eur Spine. J 2001; 10:325-33.

5. Benzel E. Biomechanics of spine stabilization. New York: Thieme, 2001.

6. Öktenoglu B, Ferreira L, Andalkar B, Özer A, Sario"glu A, Benzel E. Effects of hole preparation on screw pullout resistance and insertional torque: a biomechanical study. J Neurosurg. 2006; 94:91-6.

7. Van Ooterwyck K, Duyck J, Vander S, Van der Perre G, De Cooman M, Lievens S.,et al. I. The influence of bone mechanical properties and implant fixation upon bone loading around implants. Clin Oral Impl Res. 1998; 9:407-18.

8. Brownwe D, Jupiter J, Levine A, Trafton P. Skeletal Trauma. Philadelphia: Saunders; 1998.

9. Wagner H. Die Einbettung von Metallschrauben im Knochen und die Heilungsvorgänge des Knochengewebes unter dem Einfluss der stabilen Osteosynthese. Langenbecks Arch Klin Chir. 1963; 305:28-40.

10. Abrahão $F$, Shimano A, Defino $H$. Estudo da influência da técnica de preparação dos pedículos vertebrais na resistência ao arrancamento dos implantes. COLUNA/COLUMNA. 2003; 2:111-7.

11. Boos N, Webb JK. Pedicle screw fixation in spinal disorders: a European view. Eur Spine J. 1997; 6:2-18.
12. Brantley AG, Mayfield JK, Koeneman JB, Clark KR. The effects of pedicle screw fit. An in vitro study. Spine. 1994; 19:1752-8.

13. Carmouche JJ, Molinari RW, Gerlinger T, Devine J, Patience T. Effects of pilot hole preparation technique on pedicle screw fixation in different regions of the osteoporotic thoracic and lumbar spine. J Neurosurg Spine. 2005; 3:364-70.

14. Daftari TK, Horton WC, Hutton WC. Correlations between screw hole preparation, torque of insertion, and pullout strength for spinal screws. J Spinal Disord. 1994; 7:139-45.

15. Skinner R, Maybee J, Transfeldt E, Venter R, Chalmers W. Experimental pullout testing and comparison of variables in transpedicular screw fixation. A biomechanical study. Spine. 1990; 15:195-201.

16. Smith SA, Abitbol JJ, Carlson GD, Anderson DR, Taggart KW, et al. The effects of depth of penetration, screw orientation, and bone density on sacral screw fixation. Spine. 1993;18:1006-10.

17. Schtzker J, Horne J. The reaction of cortical bone to compression by screw threads. Clin Orthop Relat Res. 1975; (111):263-5

18. Kwok AW, Finkelstein JA, Woodside T, Hearn TC, Hu RW Insertional torque and pull-out strengths of conical and cylindrical pedicle screws in cadaveric bone. Spine. 1996; 21:2429-34.

19. George DC, Krag MH, Johnson CC, Van Hal ME, Haugh LD, Grobler LJ. Hole preparation techniques for transpedicle screws. Effect on pull-out strength from human cadaveric vertebrae. Spine. 1991; 16:181-4.

20. Ronderos JF, Jacobowitz R, Sonntag VK, Crawford NR, Dickman CA. Comparative pull-out strength of tapped and untapped pilot holes for bicortical anterior cervical screws. Spine. 1997; 22:167-70.

21. Phillips J, Rahn B. Comparison of compression and torque measurements of self- tapping and pre-tapped screw. Plas Reconstr Surg. 1989; 83:447-56.

22. Koranyi E, Bowman C, Knecht C. Holding power of orthopedic crews in bone. Clin Orthop Relat Res. 1970; (72):283-6. 